\title{
The use of glucagon-like peptide-1 receptor agonists and bone fractures: A meta-analysis of randomized clinical trials
}

\author{
Guillaume MABILLEAU, Aleksandra MIECZKOWSKA, Daniel CHAPPARD \\ GEROM-LHEA, Institut de Biologie en Santé, LUNAM Université d'Angers, Angers, France
}

\author{
Correspondence \\ Guillaume Mabilleau, GEROM- \\ LHEA, Institut de biologie en santé, \\ 4 rue larrey, 49933 Angers Cedex, \\ France. \\ Tel: $+33(0) 244688455$ \\ Fax: $+33(0) 244688451$ \\ Email: guillaume.mabilleau@univ- \\ angers.fr
}

\begin{abstract}
Background: Patients suffering of type 2 diabetes mellitus (T2DM) present a higher risk of suffering from bone fracture independently of the antidiabetic medications usage. Furthermore, anti-diabetic medications could directly affect bone metabolism. Recently, the use of dipeptidyl peptidase-4 inhibitors has been associated with a lower rate of bone fracture. The aim of the present meta-analysis was to assess whether patients with T2DM treated with GLP-1 receptor agonists (GLP-1Ra) present a lower incidence of bone fracture as compared with other anti-diabetic drugs.
\end{abstract}

Methods: A search on Medline, Embase, www.clinicaltrials.gov and handsearch for randomized clinical trials of T2DM treated with either a GLP-1Ra or another anti-diabetic drug for duration of $\geq 24$ weeks was conducted by two authors independently.

Results: Although we identified 28 eligible studies, only 7 trials reported occurrence of at least a bone fracture in one arm of the trial. The total number of fractures was 19 (13 and 6 with GLP-1Ra and comparator, respectively). The pooled Mantel-Haenszel odd ratio for GLP-1Ra was 0.87 $(95 \% \mathrm{Cl} 0.37-2.03, \mathrm{p}=0.739)$ in trials versus other anti-diabetic agents.

Conclusions: Our study failed to provide significant evidence that GLP-1Ra therapy in T2DM is associated with a decrease in the rate of occurrence of bone fracture.

Keywords: Bone fractures, GLP-1Ra, Meta-analysis, Type 2 diabetes mellitus.

Significant findings of the study: Treatment with GLP-1Ra was not associated with any modifications in the occurrence of bone fractures in type 2 diabetes mellitus.

What this study adds: The study strengthened our understanding of the drug safety profile of GLP-1Ra, such as exenatide and liraglutide, and our knowledge on how the incretin-based therapies in type 2 diabetes mellitus might affect bone metabolism.

\section{Introduction}

Glucagon-like peptide-1 (GLP-1) is produced and secreted from L-cells, an open-type intestinal epithelial endocrine cell, following the entry of nutrients in the gut lumen. GLP-1 is a member of the incretin family and as such, upon its binding to the GLP-1 receptor expressed at the surface of pancreatic $\beta$ cells, potentiates glucose- dependent insulin secretion from pancreatic islet 1. Several studies have demonstrated that GLP-1 secretion and action are reduced in T2DM and Vilsbøll et al. demonstrated that infusion of GLP-1 at supraphysiological doses in T2DM patients restored insulin levels comparable to non-diabetic controls. ${ }^{2-4}$ However, the rapid inactivation of native GLP-1 by the dipeptidyl 
peptidase 4 (DPP-4) precluded its use in a therapeutical manner. As such, two strategies have been employed to target the GLP-1 pathway and consisted in the use of DPP-4 inhibitors or GLP-1 receptor agonists (GLP-1Ra). Both strategies have now been approved in the treatment of type 2 diabetes mellitus (T2DM).

Patients suffering from T2DM have a high risk of developing bone fractures. ${ }^{5,6}$ Consequences of such complications are dramatic, often require surgery and may result in deformities, loss of independence or death. The mechanism leading to bone fracture in T2DM has yet to be determined but several factors may predispose T2DM patients to such complications including falls secondary to hypoglycaemia, diabetes complications, oral anti-diabetic drugs (such as thiazolidinediones) or increased advanced glycation end products production that negatively impact on the quality of the bone matrix. In rodent models, deletion of the GLP-1R has been associated with osteopenia and alteration of the collagen compartment of the bone matrix, suggesting a positive role of the GLP-1/GLP-1R pathway in bone metabolism. ${ }^{7,8}$ Furthermore, administration of GLP-1 or its enzyme resistant analogue exendin- 4 for three days in normal and diabetic rats results in increased trabecular bone mass and augmentation of the expression of osteoblast markers in these animals, suggesting a possible positive actions of GLP-1 on trabecular bone .9,10 Recently, despite no modifications in the postprandial markers of bone resorption in patients treated with the oral DPP-4 inhibitor vildagliptin, the short-term use (mean duration $\sim 35$ weeks) of oral dipeptidyl peptidase 4 (DPP4) inhibitors has been associated with a lower risk of bone fracture as compared with a placebo or metformin, sulfonylureas, thiazolidinediones or alpha-glucosidase inhibitors. ${ }^{11,12}$ As DPP-4 inhibitors prolonged the action of endogenous GLP-1, one could wonder whether GLP-1 might participate in the mechanism of reducing the occurrence of bone fracture observed in DPP-4 inhibitortreated patients. However, to date no data have been reported on the incidence of bone fracture in T2DM patients treated with long acting GLP-1Ra.

The aim of the present study was to assess the incidence of bone fracture in patients treated with GLP-1Ra in T2DM based on data published from randomized clinical trials. Our analysis showed that the incidence of fractures in T2DM patients treated with GLP-1Ra was not significantly different as compared with other anti-diabetic drugs.

\section{Methods}

We followed the PRISMA guideline in preparation and report of this meta-analysis.

\section{Data sources and searches}

A search for "exenatide", "liraglutide", "taspoglutide", "albiglutide", "lixisenatide", "dulaglutide" and "semaglutide" was performed in Medline and Embase for randomized clinical trials up to December 1, 2012. Publications in English language only were reviewed for this meta-analysis. Completed but still unpublished trials were identified through a search on www.clinicaltrials.gov website. A hand-search was also performed in the personal bibliography of the authors to identify any missing study from electronical searches.

\section{Study selection}

Two reviewers (GM and $A M$ ) independently screened abstracts according to the inclusion criteria. Full-text were reviewed when inclusion criteria could not be met solely on information provided in the abstract. Any discrepancies were resolved by consensus between the two reviewers referencing the original article.

\section{Data extraction and quality assessment}

A meta-analysis was performed including all trials with duration $\geq 24$ weeks, enrolling patients with T2DM and comparing GLP-1Ra with placebo or other active drugs. This duration was chosen based on the fact that modifications of bone microarchitecture and/or quality might need several weeks to occur. The quality of trials was assessed on some parameters as proposed by Jadad et al ${ }^{13}$. Participant baseline characteristics of the included studies were extracted and differences in baseline characteristics between groups, description of treatment allocation, intention-to-treat and drop out analysis were used to assess the quality of the study. The principal outcome was the effect of GLP-1Ra on the incidence of bone fractures reported as serious adverse events. Predefined separate analyses were performed for trials with different GLP-1Ra.

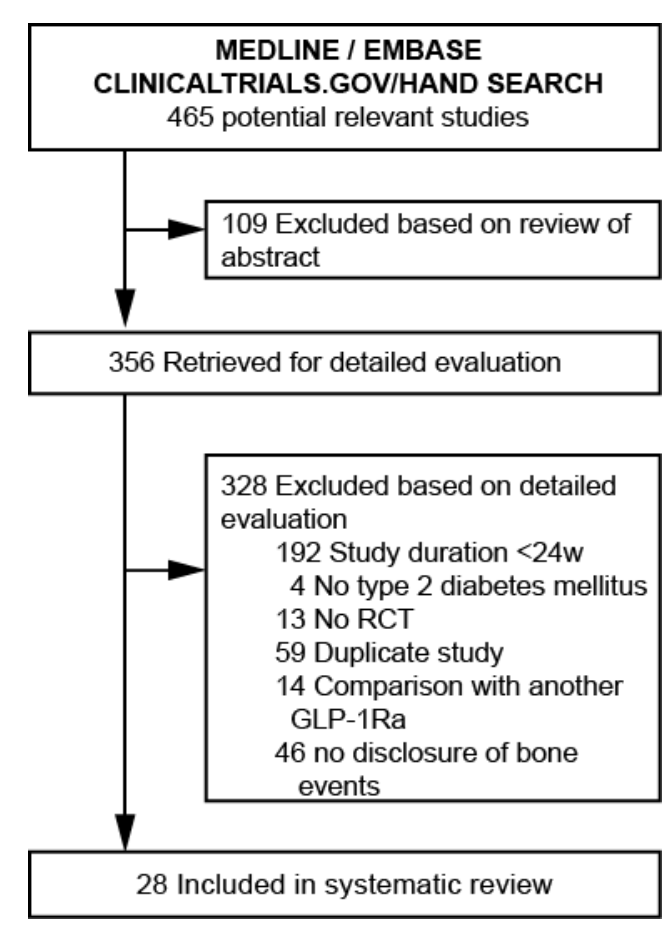

Figure 1: Study design

\section{Data synthesis and analysis}


Heterogeneity was assessed by using $I^{2}$ statistics. Data were combined using a random-effects model. The random-effects model was chosen because the validity of heterogeneity test can be limited with a small number of studies.

Table 1: Description of the quality of randomized controlled trials included in the study

\begin{tabular}{|c|c|c|c|c|c|c|}
\hline \multirow{2}{*}{ Study } & \multicolumn{3}{|c|}{ Description of } & \multirow{2}{*}{$\begin{array}{l}\text { Reporting } \\
\text { drop-out }\end{array}$} & \multirow{2}{*}{$\begin{array}{c}\text { Intention-to- } \\
\text { treat }\end{array}$} & \multirow{2}{*}{$\begin{array}{c}\text { Overall quality } \\
\text { of the study }\end{array}$} \\
\hline & Randomization & Allocation & Blinding & & & \\
\hline Buse, $2011^{14}$ & $\mathrm{~A}$ & $\mathrm{~A}$ & $\mathrm{~A}$ & $\mathrm{~A}$ & Yes & High \\
\hline Nauck, $2013^{15}$ & A & $A$ & A & A & Yes & High \\
\hline Garber, $2011^{16}$ & A & $A$ & $A$ & A & Yes & High \\
\hline Nauck, $2013^{15}$ & $A$ & $A$ & $A$ & A & Yes & High \\
\hline NCT00935532 ${ }^{1 /}$ & NA & NA & NA & A & NA & Poor \\
\hline Gallwitz, $2011^{18}$ & $A$ & NA & NA & $A$ & Yes & Medium \\
\hline Pratley, $2011^{19}$ & $A$ & $A$ & $A$ & $A$ & $A$ & High \\
\hline
\end{tabular}

A: adequately reported, NA: non-adequately reported.

The Begg adjusted rank correlation test and funnel plot were reported to assess publication bias although these tests have a low statistical power when the number of included studies is small. ${ }^{20}$ The meta-analysis was reported according to the PRISMA guideline. ${ }^{21}$ MantelHaenszel odds ratio (MH-OR) with $95 \% \quad \mathrm{Cl}$ was calculated excluding trials with no event. All analysis was performed using Comprehensive meta-analysis version 2 (Biostat, Englewood, NJ) and Systat 13 statistical softwares (Systat, San José, CA).

\section{Results}

With our search, we identified a total of 28 eligible studies (Figure 1). Of these 28 studies, 21 studies reported no fracture events in any of the treatment arm and 7 studies reported at least the occurrence of a bone fracture in one of the treatment arm. The meta-analysis was performed on 7 trials (2983 and 1246 patients treated respectively with a GLP-1Ra or comparator; mean duration of study of 67.4 weeks). Quality of the included studies is reported Table 1. Five studies were considered as high quality, one as medium quality and one as poor quality. None of the patient characteristics including percentage of women were different at baseline between GLP-1Ra and comparator arms.

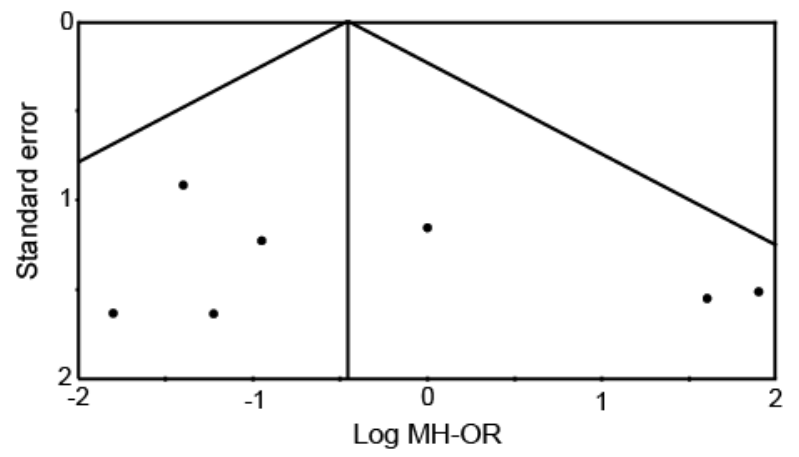

Figure 2: Funnel plot of all sub-studies on the effects of GLP-1Ra on bone fracture incidence.
Of these 7 trials, 2 were placebo-controlled, 2 were performed against the sulfonylurea glimepiride, 2 were performed vs. insulin formulations (one against insulin glargine, the other against premixed insulin aspart) and 1 was performed vs. sitagliptin (Table 2). Age, BMl and $\mathrm{HbA} 1 \mathrm{C}$ at baseline were similar in GLP-1Ra and comparator groups (Table 2). The mean age of participants was $56.4 \pm 1.9$ years, the mean BMI was $32.4 \pm 4 \mathrm{~kg} / \mathrm{m}^{2}$ and mean $\mathrm{HbA1c}$ was $8.3 \pm 0.2 \%$. The Begg adjusted correlation test (Kendall $\tau-0.047, p=0.44$ ) indicated no major publication bias. The funnel plot is represented Figure 2 and does not show any sign of publication bias. $\mathrm{I}^{2}$ test for heterogeneity suggested the use of a random-effects model.

The total number of bone fracture was 19 (13 and 6 with GLP-1Ra and comparator, respectively) for a total of 4229 patients (2983 and 1246 in the GLP-1Ra and comparator groups respectively). The pooled $\mathrm{MH}-\mathrm{OR}$ for GLP-1Ra was 0.87 (95\% Cl 0.37-2.03, p=0.739) (Figure 3). MH-OR for GLP-1Ra was 0.26 (0.05-1.33, $p=0.106)$ and $0.68(0.13-3.48, p=0.642)$ in trials vs. placebo and sulfonylurea respectively. MH-OR for GLP-1Ra vs. insulin formulations was $5.84(95 \% \mathrm{Cl} 0.7-48.737, \mathrm{p}=0.103)$. We also computed the MH-OR for GLP1Ra depending on the follow-up time. MH-OR for study $<52$ weeks was 2.45 (95\% Cl $0.57-10.49, \mathrm{p}=0.227$ ) and $\mathrm{MH}-\mathrm{OR}$ for study $\geq 52$ weeks was $0.37(95 \% \mathrm{Cl} 0.12-1.15, \mathrm{p}=0.087)$. In order to assess whether the study quality could be a cofounding factor, $\mathrm{MH}-\mathrm{OR}$ was stratified on either high or poor/medium qualities. MH-OR for poor/medium quality studies was $5.79(95 \% \mathrm{Cl} 0.69-48.45, \mathrm{p}=0.105)$ and the $\mathrm{MH}-\mathrm{OR}$ for high quality studies was $0.36(95 \% \mathrm{Cl} 0.12$ $1.05, p=0.062)$. An adjusted MH-OR was also calculated after adjustment for changes in $\mathrm{HbA1c}$. The adjusted $\mathrm{MH}$ OR was $0.88(95 \%$ Cl $0.69-1.154, p=0.157)$. No significant differences were observed between the different GLP-1Ra. 
Table 2: Characteristics of randomized controlled trials of GLP-1Ra with bone fractures

\begin{tabular}{|c|c|c|c|c|c|c|c|c|c|}
\hline Study & Drugs & $\begin{array}{c}\text { Number of } \\
\text { participants } \\
\text { (GLP-1Ra/ } \\
\text { Comparator) }\end{array}$ & $\begin{array}{c}\text { Number of bone } \\
\text { fractures } \\
\text { (GLP-1Ra/ } \\
\text { Comparator) }\end{array}$ & $\begin{array}{l}\text { Study } \\
\text { duration } \\
\text { (weeks) }\end{array}$ & $\begin{array}{l}\text { Age at } \\
\text { baseline } \\
\text { (years) }\end{array}$ & $\begin{array}{l}\text { Body mass } \\
\text { index at } \\
\text { baseline }\end{array}$ & $\begin{array}{c}\mathrm{HbA1c} \text { at } \\
\text { baseline } \\
\% \\
\text { (mmol/mol) }\end{array}$ & $\begin{array}{c}\text { Number of } \\
\text { women (GLP- } \\
1 \mathrm{Ra} / \\
\text { Comparator) } \\
\%\end{array}$ & $\begin{array}{c}\text { Changes in } \\
\text { HbA1c } \\
\text { (GLP-1Ra/ } \\
\text { Comparator) } \\
\%\end{array}$ \\
\hline Buse, $2011^{14}$ & Exenatide BID/Placebo & $137 / 122$ & $0 / 1$ & 30 & 59 & 33.5 & $8.4(68)$ & $49 / 36$ & $-1.7 /-1.0$ \\
\hline Nauck, $2013^{15}$ & Liraglutide/Placebo & $724 / 121$ & $3 / 2$ & 104 & 57 & 31.0 & $8.4(68)$ & $42 / 40$ & $-0.5 / 0.3$ \\
\hline Garber, $2011^{16}$ & Liraglutide/Glimepiride & $497 / 97$ & $2 / 1$ & 104 & 53 & 33.0 & $8.2(66)$ & $42 / 43$ & $-0.5 /-0.5$ \\
\hline Nauck, $2013^{15}$ & Liraglutide/Glimepiride & $724 / 242$ & $3 / 1$ & 104 & 57 & 30.9 & $8.4(68)$ & $52 / 47$ & $-0.7 /-0.3$ \\
\hline NCT00935532 ${ }^{17}$ & Exenatide LAR/Ins. Glargine & $215 / 212$ & $2 / 0$ & 26 & 57 & $\mathrm{~N} / \mathrm{A}$ & $8.5(69)$ & $34 / 30$ & $-1.1 /-0.7$ \\
\hline Gallwitz, $2011^{18}$ & Exenatide BID/Ins. Aspart & $247 / 233$ & $3 / 0$ & 26 & 57 & 33.2 & $7.9(63)$ & $40 / 44$ & $-1.0 /-1.1$ \\
\hline Pratley, $2011^{19}$ & Liraglutide/Sitagliptin & $439 / 219$ & $0 / 1$ & 52 & 55 & 32.8 & $8.4(68)$ & $48 / 45$ & $-1.4 /-0.9$ \\
\hline
\end{tabular}

N/A: not available 


\section{Discussion}

Bone fractures are not among usual end points considered in choosing a blood-glucose lowering agents. However, the choice of anti-diabetic medications might influence and favor bone fracture as observed with thiazolidinediones. ${ }^{22}$ The results of the present metaanalysis suggest that the use of GLP-1Ra was not associated with a reduce incidence of bone fracture as compared with other anti-diabetic drugs. However, the results of this analysis should be considered with caution as regards with the low number of trials reporting evidence of fractures.

Bone fractures were not the principal end-points of the retrieved studies and as such often disclose as a serious adverse events although this represents probably only a fraction of all fractures. Non-serious adverse events were not considered in the present study as they were often incomplete and reported events with an incidence superior to $5 \%$. Furthermore, the Begg adjusted rank correlation test and funnel plot used in the present study to investigate publication bias have a low statistical power when the number of included studies is small and as such although these statistical tools suggested no publication bias, we cannot totally rule out this artifact.

Another limitation in our study is the lack of data on bone status (bone mineral density, microarchitecture, bone quality) and calcium and phosphorus metabolism at baseline that could have highlighted differences between included patients and could have resulted in the observed effects.

$\begin{array}{lrrrrr}\text { Study name } & \text { MH-OR } & \begin{array}{c}\text { Lower } \\ \text { limit }\end{array} & \begin{array}{c}\text { Upper } \\ \text { limit }\end{array} & \text { Z-value } & \text { p-value } \\ \text { Buse, 2011 } & 0,295 & 0,012 & 7,298 & -0,746 & 0,455 \\ \text { Nauck, 2013 } & 0,248 & 0,041 & 1,497 & -1,520 & 0,128 \\ \text { Garber, 2011 } & 0,388 & 0,035 & 4,320 & -0,770 & 0,441 \\ \text { Nauck, 2013 } & 1,003 & 0,104 & 9,686 & 0,002 & 0,998 \\ \text { NCT00935532 } & 4,977 & 0,238 & 104,276 & 1,034 & 0,301 \\ \text { Gallwitz, 2011 } & 6,685 & 0,343 & 130,124 & 1,254 & 0,210 \\ \text { Pratley, 2011 } & 0,166 & 0,007 & 4,085 & -1,099 & 0,272 \\ & 0,865 & 0,369 & 2,027 & -0,333 & 0,739\end{array}$

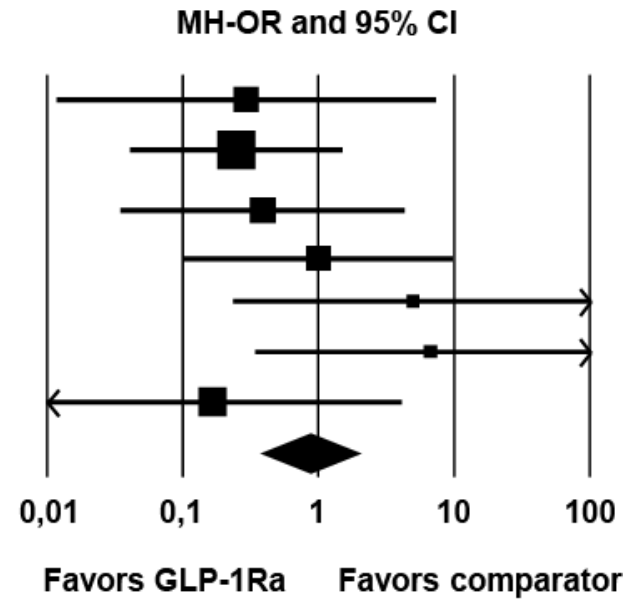

Figure 3: Subgroup analyses of $\mathrm{MH}-\mathrm{OR}(95 \% \mathrm{Cl})$ on the incidence of bone fracture in GLP-1Ra and comparator trials.

In our stratification analysis, we observed a nonsignificant increase in MH-OR for GLP-1Ra vs. insulin formulations analysis. Despite anabolic effects in bone, insulin has been previously associated with an higher risk of bone fracture in diabetes mellitus, as such one could wonder why this $\mathrm{MH}-\mathrm{OR}$ was so high. ${ }^{23-25}$ One explanation might reside in the quality of the two included studies that was defined as medium and poor. Indeed, MH-OR calculated for high quality study almost reached statistical significance $(p=0.062)$ whilst medium/poor studies were not significant with a very wide $95 \%$ confidence interval. As such it is plausible that due to the lack of description concerning allocation, randomization, blinding and intention-to-treat a bias has arisen and contributed to this higher $\mathrm{MH}-\mathrm{OR}$.

Previously, DPP-4 inhibitors were positively associated with a reduction in the incidence of bone fracture in human randomized trials as compared with other anti-diabetic drugs with a $\mathrm{MH}-\mathrm{OR}$ of 0.60 (95\% Cl 0.37-0.99, $\mathrm{p}=0.045) .^{12}$ Age, sex, BMI, duration of diabetes and $\mathrm{HbA} 1 \mathrm{c}$ at baseline were similar in this meta-analysis as compared with the present study. As GLP-1 is one of the peptides inactivated by DPP-4, one could wonder whether the reduction in bone fractures observed with short term exposure to DPP-4 inhibitors was mediated through a GLP-1-dependent mechanism. However, in the present study, we failed to highlight a significant beneficial effect of GLP-1Ra therapy on the incidence of fractures over other anti-diabetic drugs. One could wonder whether the duration of exposure to GLP-1Ra was long enough to allow bone "effects". In the present meta-analysis, the mean duration of exposure to GLP-1Ra was almost twice as those with DPP-4 inhibitors (67.4 weeks vs. 35 weeks) where a significant reduction in bone fracture has been evidenced. ${ }^{12}$ Nevertheless, stratification depending on follow-up time highlighted that longer studies ( $\geq 52$ weeks) presented with an $\mathrm{MH}-\mathrm{OR}$ lower than studies with a follow-up period $<52$ weeks and almost reach statistical significance. 
GLP1Ra and bone fractures

A major difference between DPP-4 inhibitors and GLP-1Ra lies in weight loss. Indeed, weight loss is modest in most patients taking GLP-1Ra (-2.6 \pm 0.8 $\mathrm{kg}$ as an average in our study) whereas it is neutral in patients with DPP-4 inhibitors. ${ }^{26,27}$ Weight loss after the age of 50 has previously been associated with an increased risk of fracture in overweight and obese individuals. ${ }^{28,29}$ As the mean age of participants at baseline was $56.4 \pm 1.9$ years old and the mean BMI of $32.4 \pm 4 \mathrm{~kg} / \mathrm{m}^{2}$, it is plausible that beneficial effect of GLP-1 signaling in bone might be underestimated due to weight loss.

Furthermore, another difference between GLP-1Ra and DPP-4 inhibitors lies in the most common treatment-emergent adverse events: nausea, vomiting and diarrhea were often encountered with GLP-1Ra but not with DPP-4 inhibitors. ${ }^{26}$ As such, it is also plausible that due to these adverse events, malabsorption of mineral and nutrients may have occurred and negatively balanced the positive effects of GLP-1Ra in bone physiology. Also, in rodents the GLP-1 receptor is expressed on C-cells of the thyroid gland and GLP-1Ra have been shown to promote calcitonin secretion and associated with the development of C-cell tumors in these animals. ${ }^{30}$ However such effects has not yet been observed in humans up to two-years treatments. ${ }^{31}$ As such regarding the role of calcitonin in bone biology, it is plausible that the observed anabolic effects of GLP1 on bone in rodent models might be the results of direct bone cells- and indirect calcitonin-mediated events. Another explanation to understand the discrepancy between DPP-4 inhibitors and GLP$1 \mathrm{Ra}$ may reside in the bone effects of other molecules that are physiologically inactivated by DPP-4. Indeed, receptors for GIP and glucagon-like peptide 2 (GLP-2) have been evidenced at the surface of bone cells and these two molecules have been implicated in the control of bone remodeling. ${ }^{32-}$

${ }^{34}$ As such, it is plausible that reduction of bone fracture occurrence observed with DPP-4 inhibitors might results from a complex network of interactions between several hormones that are physiologically inactivated by DPP-4 rather than only the rise in GLP-1.

In conclusion, the present meta-analysis failed to provide significant evidence that the use of GLP. 1 Ra significantly reduced the occurrence of bone fracture in T2DM as compared with other antidiabetic agents. Nevertheless, with regards to the low number of data presently available, a more careful assessment of the incidence of fracture in ongoing trials with GLP-1Ra should be performed.

\section{Acknowledgments}

This study was funded by the Bioregos 2 program. The founders were not involved in the study design or results.

\section{Disclosure}

The authors have no conflict of interest to declare.

\section{References}

1. Mojsov S, Weir GC, Habener JF. Insulinotropin: glucagon-like peptide I (7-37) co-encoded in the glucagon gene is a potent stimulator of insulin release in the perfused rat pancreas. J Clin Invest. 1987; 79:616-9.

2. Nauck MA, Heimesaat MM, Orskov C, Holst JJ, Ebert R, Creutzfeldt W. Preserved incretin activity of glucagon-like peptide 1 [7-36 amide] but not of synthetic human gastric inhibitory polypeptide in patients with type-2 diabetes mellitus. J Clin Invest. 1993; 91:301-7.

3. Vilsboll T, Krarup T, Deacon CF, Madsbad S, Holst JJ. Reduced postprandial concentrations of intact biologically active glucagon-like peptide 1 in type 2 diabetic patients. Diabetes. 2001; 50:609-13. 4. Vilsboll T, Krarup T, Madsbad S, Holst JJ. Defective amplification of the late phase insulin response to glucose by GIP in obese Type II diabetic patients. Diabetologia. 2002; 45:1111-9.

5. de Liefde I, van der Klift $M$, de Laet $C E$, van Daele PL, Hofman A, Pols HA. Bone mineral density and fracture risk in type-2 diabetes mellitus: the Rotterdam Study. Osteoporos Int. 2005; 16:171320.

6. Schwartz AV, Vittinghoff $\mathrm{E}$, Bauer $\mathrm{DC}$ et al. Association of BMD and FRAX score with risk of fracture in older adults with type 2 diabetes. JAMA. 2011; 305:2184-92.

7. Mabilleau G, Mieczkowska A, Irwin N, Flatt PR, Chappard D. Optimal bone mechanical and material properties require a functional GLP-1 receptor. J Endocrinol. 2013; 219:59-68.

8. Yamada $\mathrm{C}$, Yamada $\mathrm{Y}$, Tsukiyama $\mathrm{K}$ et al. The murine glucagon-like peptide-1 receptor is essential for control of bone resorption. Endocrinology. 2008; 149:574-9.

9. Nuche-Berenguer $\mathrm{B}$, Moreno $\mathrm{P}$, Esbrit $\mathrm{P}$ et al. Effect of GLP-1 treatment on bone turnover in normal, type 2 diabetic, and insulin-resistant states. Calcif Tissue Int. 2009; 84:453-61.

10. Nuche-Berenguer B, Moreno $P$, Portal-Nunez $S$, Dapia S, Esbrit $\mathrm{P}$, Villanueva-Penacarrillo $\mathrm{ML}$. Exendin-4 exerts osteogenic actions in insulinresistant and type 2 diabetic states. Regul Pept. 2010; 159:61-6.

11. Bunck MC, Poelma M, Eekhoff EM et al. Effects of vildagliptin on postprandial markers of bone resorption and calcium homeostasis in recently diagnosed, well-controlled type 2 diabetes patients. J Diabetes. 2012; 4:181-5.

12. Monami M, Dicembrini I, Antenore A, Mannucci E. Dipeptidyl peptidase-4 inhibitors and bone fractures: a meta-analysis of randomized clinical trials. Diabetes Care. 2011; 34:2474-6.

13. Jadad AR, Moore RA, Carroll D et al. Assessing the quality of reports of randomized clinical trials: is blinding necessary? Control Clin Trials. 1996; 17:112.

14. Buse JB, Bergenstal RM, Glass LC et al. Use of twice-daily exenatide in basal insulin-treated patients with type 2 diabetes. Ann Intern Med. 2011; 154:103-12.

15. Nauck $M$, Frid $A$, Hermansen $K$ et al. Long-term efficacy and safety comparison of liraglutide, glimepiride and placebo, all in combination with 
GLP1Ra and bone fractures

metformin in type 2 diabetes: 2 -year results from the LEAD-2 study. Diabetes Obes Metab. 2013; 15:20412.

16. Garber A, Henry RR, Ratner R et al. Liraglutide, a once-daily human glucagon-like peptide 1 analogue, provides sustained improvements in glycaemic control and weight for 2 years as monotherapy compared with glimepiride in patients with type 2 diabetes. Diabetes Obes Metab. 2011; 13:348-56

17. http://clinicaltrials.gov (accessed).

18. Gallwitz B, Bohmer M, Segiet $T$ et al. Exenatide twice daily versus premixed insulin aspart 70/30 in metformin-treated patients with type 2 diabetes: a randomized 26-week study on glycemic control and hypoglycemia. Diabetes Care. 2011; 34:604-6.

19. Pratley $R$, Nauck M, Bailey $T$ et al. One year of liraglutide treatment offers sustained and more effective glycaemic control and weight reduction compared with sitagliptin, both in combination with metformin, in patients with type 2 diabetes: a randomised, parallel-group, open-label trial. Int $J$ Clin Pract. 2011; 65:397-407.

20. Sterne JA, Gavaghan D, Egger M. Publication and related bias in meta-analysis: power of statistical tests and prevalence in the literature. $J$ Clin Epidemiol. 2000; 53:1119-29.

21. Liberati A, Altman DG, Tetzlaff $\mathrm{J}$ et al. The PRISMA statement for reporting systematic reviews and meta-analyses of studies that evaluate healthcare interventions: explanation and elaboration. BMJ. 2009; 339:b2700.

22. Kahn SE, Zinman B, Lachin JM et al. Rosiglitazone-associated fractures in type 2 diabetes: an Analysis from A Diabetes Outcome Progression Trial (ADOPT). Diabetes Care. 2008; 31:845-51.

23. Lipscombe LL, Jamal SA, Booth GL, Hawker $\mathrm{GA}$. The risk of hip fractures in older individuals with diabetes: a population-based study. Diabetes Care. 2007; 30:835-41.

24. Nicodemus KK, Folsom AR, lowa Women's Health S. Type 1 and type 2 diabetes and incident hip fractures in postmenopausal women. Diabetes Care. 2001; 24:1192-7.

25. Schwartz AV, Sellmeyer DE, Ensrud KE et al. Older women with diabetes have an increased risk of fracture: a prospective study. J Clin Endocrinol Metab. 2001; 86:32-8.

26. Amori RE, Lau J, Pittas AG. Efficacy and safety of incretin therapy in type 2 diabetes: systematic review and meta-analysis. JAMA. 2007; 298:194206.

27. Inzucchi SE, Bergenstal RM, Buse JB et al. Management of hyperglycemia in type 2 diabetes: a patient-centered approach: position statement of the American Diabetes Association (ADA) and the European Association for the Study of Diabetes (EASD). Diabetes Care. 2012; 35:1364-79.

28. Jensen LB, Quaade F, Sorensen $\mathrm{OH}$. Bone loss accompanying voluntary weight loss in obese humans. J Bone Miner Res. 1994; 9:459-63.

29. Langlois JA, Mussolino ME, Visser M, Looker AC, Harris T, Madans J. Weight loss from maximum body weight among middle-aged and older white women and the risk of hip fracture: the NHANES I epidemiologic follow-up study. Osteoporos Int. 2001; 12:763-8.

30. Madsen LW, Knauf JA, Gotfredsen C et al. GLP1 receptor agonists and the thyroid: C-cell effects in mice are mediated via the GLP-1 receptor and not associated with RET activation. Endocrinology. 2012; 153:1538-47.

31. Hegedus L, Moses AC, Zdravkovic M, Le Thi T, Daniels GH. GLP-1 and calcitonin concentration in humans: lack of evidence of calcitonin release from sequential screening in over 5000 subjects with type 2 diabetes or nondiabetic obese subjects treated with the human GLP-1 analog, liraglutide. J Clin Endocrinol Metab. 2011; 96:853-60.

32. Gaudin-Audrain C, Irwin N, Mansur S et al. Glucose-dependent insulinotropic polypeptide receptor deficiency leads to modifications of trabecular bone volume and quality in mice. Bone. 2013; 53:221-30.

33. Henriksen DB, Alexandersen P, Byrjalsen I et al. Reduction of nocturnal rise in bone resorption by subcutaneous GLP-2. Bone. 2004; 34:140-7.

34. Pacheco-Pantoja EL, Ranganath LR, Gallagher JA, Wilson PJ, Fraser WD. Receptors and effects of gut hormones in three osteoblastic cell lines. BMC Physiol. 2011; 11:12. 
GLP1Ra and bone fractures 